Check for updates

Cite this: RSC Adv., 2018, 8, 162

Received 25th October 2017 Accepted 4th December 2017 DOI: $10.1039 / c 7 r a 11784 a$

rsc.li/rsc-advances

\section{The chemical redox modulated switch-on fluorescence of carbon dots for probing alkaline phosphatase and its application in an immunoassay $\dagger$}

\begin{abstract}
Pei Song, (D) Qian Liu, Ying Zhang, Wei Liu, Meng Meng, Yongmei Yin* and Rimo Xi*
Early diagnosis is an important requirement for the early treatment of diseases and reliable detection methods for biomarkers are indispensable tools for early diagnosis. However, recent innovations in analytical technology are rarely employed widely due to multiple factors including the requirement of specialized equipment and infrastructures. Hence, much attention has been focused on exploring universal nanoprobes for routine practices. Here, we report a chemical redox modulated switch-on fluorescence strategy based on versatile carbon dots (CDs) for probing alkaline phosphatase (ALP) and human IgG (a model target). The fluorescence of the CDs is initially quenched by Fe(III) and then restored by ascorbic acid, which is generated from the hydrolysis of ascorbic acid 2-phosphate (AAP) by the catalysis of ALP. Based on that, a simple "switch-on" fluorescence assay for ALP was designed and leads to a sensitivity of as low as $0.8 \mathrm{UL}^{-1}$. The proposed assay is applied in ALP sensing in human serum samples. Additionally, the application of the switch-on fluorescence strategy is successfully expanded to the immunoassay of human IgG in serum from healthy adults and patients with systemic lupus erythematosus. The developed sensing system could be devised for various target sensors and has promising potential in the field of biological analysis and clinical diagnostics.
\end{abstract}

\section{Introduction}

The development of a distinctive assay for biomarker detection provides abundant options for biology and disease diagnosis. Despite remarkable achievements in laboratories, the extensive adoption of reported technological innovations in clinic is rare. This phenomenon is due to several reasons, such as the need to introduce different equipment, infrastructures and specialized operator training, and to consider the cost and acceptance of patients. Thus, renovating the traditional routine protocols is also a feasible direction for improving the ability of detection for diagnosis., ${ }^{\mathbf{1} 2}$

It is well known that alkaline phosphatase (ALP), the membrane-bound enzyme that plays a critical role in the signal transduction and regulation of intracellular processes, ${ }^{3-6}$ can be found in various tissues of living organisms such as intestine, bone, liver, placenta, and kidney. ${ }^{7-10}$ The abnormal level of serum ALP is related to various diseases including dynamic bone disease, diabetes, breast and prostate cancer, and liver dysfunction. ${ }^{11-13}$ In addition, ALP is a widely used labeling

State Key Laboratory of Medicinal Chemical Biology, College of Pharmacy, Tianjin Key Laboratory of Molecular Drug Research, Nankai University, Tianjin 300353, China. E-mail:xirimo@nankai.edu.cn; yinyongmei@nankai.edu.cn

$\dagger$ Electronic supplementary information (ESI) available. See DOI: 10.1039/c7ra11784a enzyme for use in immunoassays, ${ }^{\mathbf{1 4}}$ DNA hybridization analysis and protein detection. ${ }^{\mathbf{1 5 , 1 6}}$ Therefore, it is of great importance to develop a convenient and sensitive method for ALP level monitoring in various diagnostic applications.

Nowadays, fluorescence nanomaterials including quantum dots (QDs), noble metal nanoclusters, and carbon dots (CDs) receive much attention because of advantages including their easy synthesis and good stability, and a lack of photobleaching. Among them, CDs possess the unique properties of low toxicity and favorable biocompatibility. ${ }^{17-22}$ Studies on CD-based chemosensors for ALP have been reported previously. Qian et al. developed a detection strategy for ALP based on a turn-off process, which was induced by the disaggregation of CDs with the assistance of copper ions and $\mathrm{PPi}^{23} \mathrm{Li}$ et al. used a fluorescence assay to detect ALP based on the inner filter effect. When in the presence of ALP, $p$-nitrophenylphosphate was transformed into $p$-nitrophenol (PNP) and induced the absorption band transition from 310 to $405 \mathrm{~nm}$, which resulted in the complementary overlap between the absorption band of PNP and the excitation band of CDs. ${ }^{24}$ Tang et al. developed a convenient assay for ALP through specific host-guest recognition based on $\beta$-cyclodextrin-modified CDs. The photoinduced electron transfer process between PNP and the CDs results in the quenching of the fluorescence of the CDs. ${ }^{25}$ Unfortunately, all of these assays are based on fluorescence 


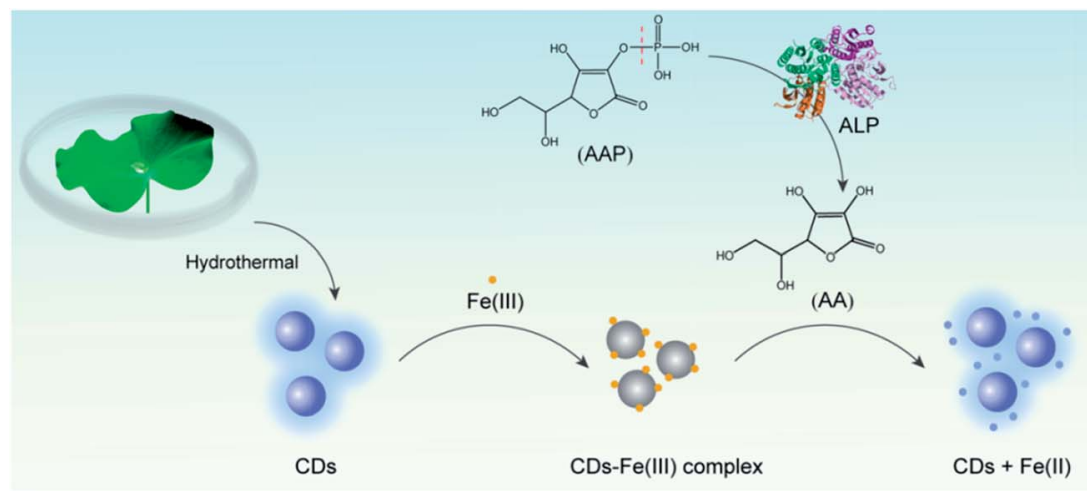

Fig. 1 Schematic illustration of the detection strategy for ALP based on the chemical redox modulated switch-on fluorescence of carbon dots.

quenching. However, such enzyme sensors do not contribute to a signal output on account of the high background signals. ${ }^{26,27}$ Additionally, the turn-off detection mode might cause an increased possibility of false positive signals due to selfquenching, solvent quenching, and so on. ${ }^{28,29}$ A "switch-on" sensing platform avoids the above disadvantage, whereas CDbased "switch-on" fluorescence assays for probing ALP have been rarely reported.

In this study, we developed a "switch-on" fluorescence assay for sensing ALP based on a simple chemical redox strategy. The fluorescence of the CDs prepared from lotus leaves is initially quenched specifically by $\mathrm{Fe}(\mathrm{III})$, and then restored by ascorbic acid (AA) generated from the hydrolysis of ascorbic acid 2phosphate (AAP) by the catalysis of ALP (Fig. 1). The recovery of the fluorescence is due to the redox reaction between AA and $\mathrm{Fe}(\mathrm{III})$, which destroys the structure of the non-fluorescent CDFe(III) complex. The "switch-on" fluorescence assay for sensing ALP was successfully applied to the determination of ALP in human serum samples. Additionally, the ALP detection strategy was successfully combined with the immunoassay to monitor human IgG (as a model). This approach provides a new pathway to determine, but is not restricted to, ALP. It is a versatile platform with the ability to sense various targets and the platform can be extended to a large range of biological analyses and clinical diagnostics.

\section{Experimental section}

\section{Materials and instruments}

Lotus leaves were obtained from Mati Lake at Nankai University and washed thoroughly before use. $\mathrm{FeCl}_{3}$ was purchased from Aladdin Chemistry Co., Ltd. (Shanghai, China). Alkaline phosphatase (ALP, from bovine intestinal mucosa) and ascorbic acid (AA) were bought from Sigma-Aldrich (St. Louis, MO, USA). Ascorbic acid 2-phosphate (AAP) was purchased from AdamasBeta (Shanghai, China). Rabbit antihuman IgG and human IgG were obtained from Solarbio Bioscience \& Technology Co., Ltd. (Beijing, China). ALP-labeled goat antihuman IgG was purchased from ImmunoReagents Inc. (Carolina, USA). Highpurity water, which was purified using a Millipore System
(Molsheim, France), was used throughout the experiments. Other chemicals were of analytical grade and used as received.

The fluorescence measurements were conducted on a Hitachi F-4600 FL spectrophotometer using a $5 / 5 \mathrm{~nm}$ slit width (Tokyo, Japan). Fourier transform infrared spectra (FT-IR) were acquired on a BRUKER Tensor 37 spectrometer using $\mathrm{KBr}$ pellets (Germany). UV-vis absorption spectra were recorded on a Shimadzu UV-1750 spectrophotometer with one pair of $10 \mathrm{~mm}$ quartz cells (Japan). The transmission electron microscopic (TEM) image was acquired on a FEI Tecnai G2 F20 microscope operating at $200 \mathrm{kV}$ (Hillsboro, USA). Particle size analysis was done using Image J software. The X-ray photoelectron spectra (XPS) were obtained out using a PE PHI-5300 ESCA system (Massachusetts, USA).

\section{Synthesis of carbon quantum dots}

CDs were simply synthesized by the hydrothermal method. Briefly, $2 \mathrm{~g}$ of lotus leaves and $10 \mathrm{~mL}$ of ultrapure water were added into a $50 \mathrm{~mL}$ Teflon-lined autoclave and heated at $200^{\circ} \mathrm{C}$ for $5 \mathrm{~h}$. After the mixture was cooled to room temperature naturally, the carbide slag was removed through centrifugation at $12000 \mathrm{rpm}$ for $10 \mathrm{~min}$. The supernatant was purified by filtration through a $0.22 \mu \mathrm{m}$ filter membrane and then diluted with ultrapure water for further use.

\section{Fluorescence quenching of the CDs by ferric ions}

Ten microliters of Fe(III) solution $(0,5,10,20,30,40,50,60,80$, $100,200,300,400,600 \mu \mathrm{M})$ was added into the CD solution $(990$ $\mu \mathrm{L}, 1 \mu \mathrm{L} \mathrm{mL}{ }^{-1}$ ), then they were mixed. After incubation at room temperature, the fluorescence emission spectra were recorded at the excitation wavelength of $320 \mathrm{~nm}$. The specificity of the CDs for sensing $\mathrm{Fe}(\mathrm{III})$ was evaluated by the interfering metal ions $\left(\mathrm{Fe}^{2+}\right.$, $\mathrm{Cu}^{2+}, \mathrm{Cd}^{2+}, \mathrm{Cr}^{3+}, \mathrm{Zn}^{2+}, \mathrm{Mn}^{2+}, \mathrm{Ni}^{2+}, \mathrm{Co}^{2+}, \mathrm{Al}^{3+}, \mathrm{Pb}^{2+}, \mathrm{Ag}^{+}, \mathrm{Hg}^{2+}, \mathrm{Mg}^{2+}$, $\mathrm{Ba}^{2+}, \mathrm{Ca}^{2+}$ ) under the same conditions. Besides, $100 \mu \mathrm{M}$ of commonly used compounds (cysteine, glycine, $\mathrm{Na}_{2} \mathrm{HPO}_{4}$ and $\mathrm{NaCl}$ ) were analyzed, with the same procedures as indicated above.

\section{Fluorescence assay for ALP}

Ten microliters of AAP $(50 \mathrm{mM})$ solution and $10 \mu \mathrm{L}$ of ALP or other proteins were added into $480 \mu \mathrm{L}$ of Tris-HCl buffer 
(10 mM, pH 7.4) and incubated for $30 \mathrm{~min}$ at $37^{\circ} \mathrm{C}$. After that, $490 \mu \mathrm{L}$ of the $\mathrm{CD}$ (Tris- $\mathrm{HCl}$ ) solution was added to make the $\mathrm{pH}$ of the mixture equal to 6.5 and then $10 \mu \mathrm{L}$ of $\mathrm{Fe}(\mathrm{III})(300 \mu \mathrm{M})$ was added for another incubation of $5 \mathrm{~min}$ at room temperature. The fluorescence spectra were recorded at the excitation wavelength of $320 \mathrm{~nm}$. In addition, several enzymes and anions that existed in the serum were selected to evaluate the specificity of the assay for ALP, including glucose oxidase (GOx), lysozyme, lactic dehydrogenase (LDH), trypsin, galactosidase (GAL), thrombin, tyrosinase (TYR), $\mathrm{Cl}^{-}$and $\mathrm{HCO}_{3}{ }^{-}$.

For ALP determination in complex biological media, $10 \mu \mathrm{L}$ of AAP (50 mM) solution and $10 \mu \mathrm{L}$ of ALP solutions with different final concentrations were added into $480 \mu \mathrm{L}$ of Tris- $\mathrm{HCl}$ buffer (10 mM, pH 7.4) containing 1\% human serum for $30 \mathrm{~min}$ at $37^{\circ} \mathrm{C}$. The followed procedure was the same as indicated above.

\section{Human IgG detection}

The developed "switch-on" fluorescence assay was applied to human IgG detection based on a sandwich immunoassay platform. 96-well plates were coated with $1 \mu \mathrm{g} \mathrm{mL} \mathrm{mL}^{-1}$ of anti-human IgG $(100 \mu \mathrm{L}$ per well $)$ at $4{ }^{\circ} \mathrm{C}$ overnight, washed 3 times with Tris-HCl buffer $(0.05 \%$ Tween 20$)$, blocked by Tris-HCl buffer (10 $\left.\mathrm{g} \mathrm{L}^{-1} \mathrm{OVA}\right)$ for $2 \mathrm{~h}$ and washed 3 times with Tris-HCl buffer (0.05\% Tween 20). Next, human IgG $\left(100 \mu \mathrm{L}, 40-4000 \mathrm{ng} \mathrm{mL}^{-1}\right)$ or the serum sample $\left(10^{5}\right.$-fold dilution) was added, incubated for $30 \mathrm{~min}$ at $37^{\circ} \mathrm{C}$ and washed 3 times. Then, ALP-labeled antihuman IgG (100 $\mu \mathrm{L}, 1$ : 200 dilution) was added, incubated for $30 \mathrm{~min}$ and washed 4 times. After this, the ALP reaction system was added and finally, the fluorescence spectra were recorded.

\section{Results and discussion}

\section{Characterization of CDs}

The as-prepared CDs were characterized using transmission electron microscopy (TEM), X-ray photoelectron spectroscopy (XPS), Fourier transform infrared spectroscopy (FT-IR), UV-vis absorption and fluorescence emission spectra. As shown in Fig. 2A, the TEM image confirms the spherical morphology and size information of the CDs (the average diameter is $2.1 \mathrm{~nm}$ ). The high resolution TEM image (inset in Fig. 2A) shows that the CDs displayed a highly crystalline structure with a $0.2 \mathrm{~nm}$ lattice parameter, which is similar to the (102) diffraction plane of graphitic $\left(\mathrm{sp}^{2}\right)$ carbon. In the XPS survey spectrum (Fig. S1A $\dagger$ ) the $\mathrm{C} \mathrm{1s,} \mathrm{N} \mathrm{1s} \mathrm{and} \mathrm{O} 1 \mathrm{~s}$ signals appear at $285 \mathrm{eV}, 400 \mathrm{eV}$ and $532 \mathrm{eV}$, respectively. The elemental composition of the CDs was analyzed and confirmed the presence of $72.48 \% \mathrm{C}, 12.95 \% \mathrm{~N}$ and $14.58 \% \mathrm{O}$. The high-resolution XPS spectrum of $\mathrm{C} 1 \mathrm{~s}$ (Fig. S1B $\dagger$ ) shows three peaks at $284.6 \mathrm{eV}, 285.4 \mathrm{eV}$ and $288.0 \mathrm{eV}$, which are assigned to $\mathrm{C}-\mathrm{C}, \mathrm{C}-\mathrm{N} / \mathrm{C}-\mathrm{O}$ and $\mathrm{C}=\mathrm{O}$, respectively. The $\mathrm{N} 1 \mathrm{~s}$ peak at $399.8 \mathrm{eV}$ (Fig. $\mathrm{S} 1 \mathrm{C} \dagger$ ) reveals the presence of $\mathrm{C}-$ $\mathrm{N}-\mathrm{C}$. The two peaks at $531.3 \mathrm{eV}$ and $532.5 \mathrm{eV}$ in $\mathrm{O} 1 \mathrm{~s}$ spectrum (Fig. S1D $\dagger$ ) are associated with the $\mathrm{C}=\mathrm{O}$ and $\mathrm{C}-\mathrm{OH} / \mathrm{C}-\mathrm{O}-\mathrm{C}$ bands. ${ }^{30}$ In addition, the functional groups on the surface of the CDs were further characterized using an FT-IR spectrum (Fig. S2 $\dagger$ ). It exhibits the stretching vibrations of $\mathrm{O}-\mathrm{H}$ and $\mathrm{N}-\mathrm{H}$ (3423 $\left.\mathrm{cm}^{-1}\right), \mathrm{C}-\mathrm{H}$ stretching vibrations $\left(2966 \mathrm{~cm}^{-1}\right), \mathrm{C}-\mathrm{N}$ stretching vibrations $\left(2360 \mathrm{~cm}^{-1}\right)$ and $\mathrm{C}=\mathrm{O}$ stretching vibrations $\left(1666 \mathrm{~cm}^{-1}\right)$. Besides, the peak at $1632 \mathrm{~cm}^{-1}$ could be identified as the stretching vibrations of $\mathrm{C}-\mathrm{O}$ and the bending vibrations of $\mathrm{N}-\mathrm{H} .{ }^{31}$ The peak at $1400 \mathrm{~cm}^{-1}$ is attributed to $\mathrm{C}-\mathrm{N}$, $\mathrm{N}-\mathrm{H}$, and -COO groups. ${ }^{32}$ The bands in the range of 1043$1120 \mathrm{~cm}^{-1}$ are assigned to the stretching and bending vibration of $\mathrm{C}-\mathrm{O}$ bonds. The $\mathrm{UV}$-vis absorption and fluorescence emission spectra of the aqueous dispersion of the CDs are presented in Fig. 2B. It is seen that the resultant CDs show two weak absorption peaks at $242 \mathrm{~nm}$ and $320 \mathrm{~nm}$ which are ascribed to the $\pi-\pi^{*}$ transition of $\mathrm{C}=\mathrm{C}$ bonds and $\mathrm{n}-\pi^{*}$ transition of $\mathrm{C}=\mathrm{O}$ bonds in the CDs. ${ }^{33,34}$ The emission spectrum shows that a strong emission with a maximum emission wavelength at $412 \mathrm{~nm}$ could be observed under excitation at $320 \mathrm{~nm}$. Strong blue photoluminescence was seen under UV light (inset in Fig. 2B). Besides, the fluorescence spectra depicted an excitation dependent behavior. With an increase in excitation wavelength from $290 \mathrm{~nm}$ to $380 \mathrm{~nm}$, the maximum emission shifted from $407 \mathrm{~nm}$ to $446 \mathrm{~nm}$ (Fig. S3†). The strongest emission spectrum was observed at an excitation wavelength of $320 \mathrm{~nm}$. The excitation-dependent fluorescence behavior of the CDs is due to the different sizes and surface states of the nanoparticles, ${ }^{35}$ as in the case of graphene quantum dots and most of the luminescent carbon dots. ${ }^{36}$

\section{Fluorescence response of the CDs and mechanism analysis}

As it has been reported that metal ions can be used to coordinate to the fluorescence indicator to form a chemosensing
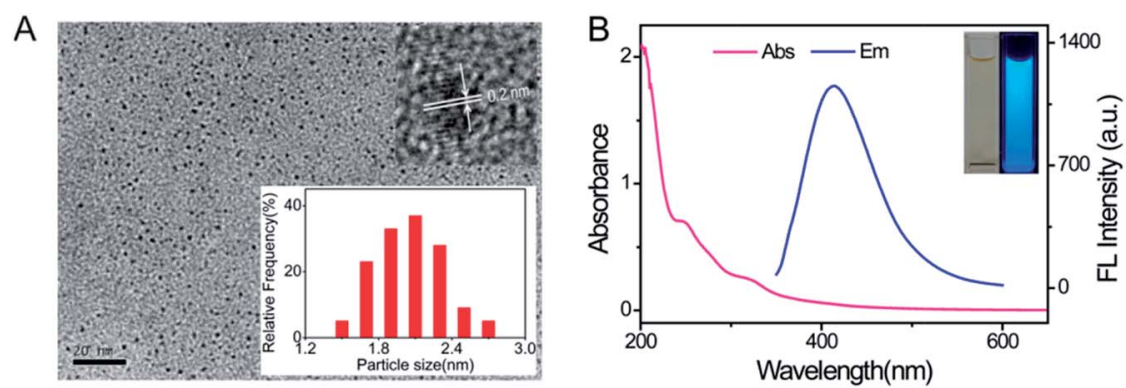

Fig. 2 (A) TEM and HRTEM (inset) images of the carbon dots and the size distribution of the diameters of the as-prepared CDs (inset). (B) UV/vis absorption spectra and emission spectra of the CDs at the excitation wavelength of $320 \mathrm{~nm}$. Inset: photographs of the CDs under visible light (left) and a UV beam of $365 \mathrm{~nm}$ (right). 
ensemble, ${ }^{37-39}$ we assessed the influence of sixteen metal cations and four compounds on the fluorescence of the CDs in this work. The introduction of the ions except with Fe(III) leads to an ignorable influence on the fluorescence of the CDs (Fig. S4 $\dagger$ ). Also, no significant change was observed with the addition of the four compounds, whereas the presence of $\mathrm{Fe}$ (III) has a remarkable impact on the fluorescence intensity with a distinct quenching effect (Fig. 3A). According to the results of the XPS and FT-IR, plentiful hydroxyl groups exist on the surface of the CDs. Furthermore, the electronegativity of oxygencontaining groups is higher than that of nitrogen-containing groups. ${ }^{40}$ Thus, we deduce that the fluorescence quenching of the CDs by $\mathrm{Fe}(\mathrm{III})$ arises from the coordination interactions between $\mathrm{Fe}$ (III) and the hydroxyl groups on the surface of the CDs. To get insight into the quenching mechanism, we measured the fluorescence lifetime to investigate the excited states of the CDs in the absence/presence of Fe(III). The fluorescence decay curves were found to fit well to a classical threeexponential function (Table $\mathrm{S} 1 \dagger$ ). In comparison to the CDs alone, the $\mathrm{CD} / \mathrm{Fe}(\mathrm{III})$ complex shows a negligible change in the lifetime (Fig. 3B). The virtually identical fluorescence lifetimes implies that the fluorescence quenching obeys a simple static mechanism. According to the above analysis, the fluorescence quenching of the CDs should be attributed to the formation of the non-fluorescent complex, which is based on the coordination interactions between Fe(III) and the hydroxyl groups on the surface of the CDs, and it is a static quenching process.

Fig. 3A exhibits the fluorescence emission spectra of differently treated CDs and shows that their fluorescence could be quenched by $\mathrm{Fe}(\mathrm{III})$. However, when AA was introduced into the
$\mathrm{CD} / \mathrm{Fe}(\mathrm{III})$ system, the quenched fluorescence was restored. In a control experiment, no change in the fluorescence intensity was observed with the individual addition of AA to the CD solution. Meanwhile, there was no detectable fluorescence when the AA solution was introduced to the Fe(III) solution. Thus, we suggest that the fluorescence restoring results from the redox reaction between $\mathrm{AA}$ and $\mathrm{Fe}(\mathrm{III})$ in the $\mathrm{CD} / \mathrm{Fe}(\mathrm{III})$ complex. To further confirm the fluorescence restoring principle, the theoretical stability constant and fluorescence lifetime were investigated. As we know, $\mathrm{Fe}(\mathrm{II})$ is produced from the redox reaction between $\mathrm{AA}$ and $\mathrm{Fe}(\mathrm{III})$. The theoretical stability constant for the complex of hydroxide and Fe(III) is $1.00 \times 10^{11}$, whereas that of hydroxide and Fe(II) is lower $\left(3.16 \times 10^{4}\right)$, which indicates that the generated $\mathrm{Fe}$ (II) may be separated from the surface of the CDs and would turn on the fluorescence. Additionally, the fluorescence decay data show that the lifetime of the resulting $\mathrm{CD} / \mathrm{Fe}(\mathrm{III}) / \mathrm{AA}$ mixture is $9.62 \mathrm{~ns}$ (Fig. 3B and Table $\mathrm{S} 1 \dagger)$, which is similar to the CDs and $\mathrm{CD} / \mathrm{Fe}(\mathrm{III})$ complex. This result suggests that the whole quenching/recovery of the fluorescence obeys a static mechanism and the destruction of the structure of the non-fluorescent $\mathrm{CD} / \mathrm{Fe}$ (III) complex induces the fluorescence recovery. Therefore, all of the above results demonstrate that the oxidation/reduction modulated by $\mathrm{Fe}(\mathrm{III}) /$ AA results in the structural construction/destruction of the non-fluorescent $\mathrm{CD} / \mathrm{Fe}$ (III) complex, leading to the fluorescence quenching/recovery.

We further evaluated the feasibility of the CDs as a fluorescent sensor for $\mathrm{Fe}(\mathrm{III})$ and $\mathrm{AA}$ detection by adding varying the concentration of the target. Fig. 3C shows the fluorescence emission spectra of the CDs when adding different amounts of
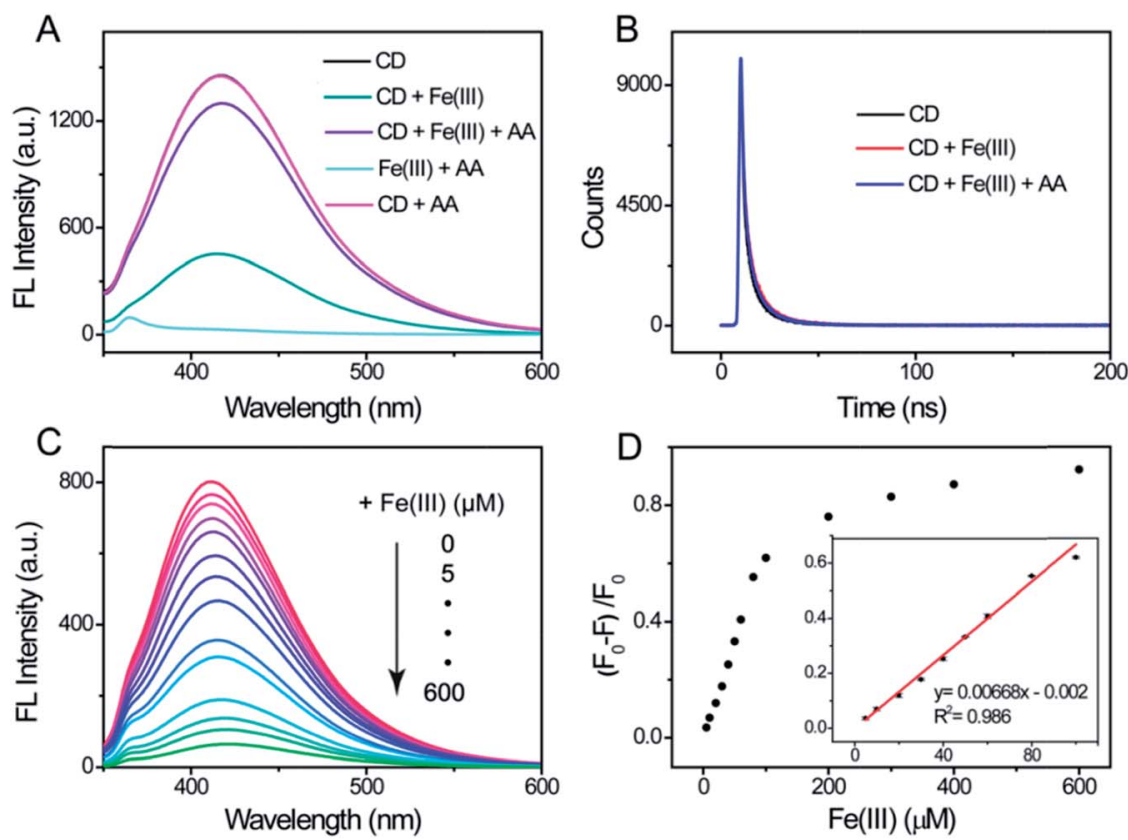

Fig. 3 (A) Fluorescence emission spectra of the CDs under different conditions. (B) Fluorescence decay curves of the CDs in the absence and presence of Fe(III) and AA upon excitation at $320 \mathrm{~nm}$. (C) Fluorescent emission spectra of the CDs upon the addition of various amounts of Fe(III). (D) The dependence of $\left(F_{0}-F\right) / F_{0}$ on the concentration of Fe(III). Inset: the linear fitting curve between $\left(F_{0}-F\right) / F_{0}$ and Fe(III) concentration within the range of $5-100 \mu \mathrm{M}$. 
$\mathrm{Fe}(\mathrm{III})$. It is found that the fluorescence of the CDs is significantly quenched by $\mathrm{Fe}$ (III) ions and the corresponding fluorescence intensity decreases obviously with the increase in the amount of $\mathrm{Fe}(\mathrm{III})$. The fluorescence intensity decreases by $92 \%$ of the original value when the concentration of $\mathrm{Fe}(\mathrm{III})$ is $600 \mu \mathrm{M}$ (Fig. 3D). A good linear relationship was obtained between the fluorescence quenching efficiency and the concentration of $\mathrm{Fe}(\mathrm{III})$ in the range of 5 to $100 \mu \mathrm{M}$ and the limit of detection for $\mathrm{Fe}(\mathrm{III})$ is $1.09 \mu \mathrm{M}$. The sensing performance of the CD-Fe(III) system for the detection of AA was evaluated by the addition of different concentrations of AA. The fluorescence intensity of the system could be gradually recovered upon the addition of AA (Fig. S5A $\dagger$ ). The degree of the fluorescence recovery is directly related to the concentration of AA. A good linear relationship between the fluorescence intensity and AA concentration was obtained in the range of 0.5 to $200 \mu \mathrm{M}$ (Fig. S5B $\dagger$ ).

\section{Assessment of the detection strategy for ALP based on CDS and AAP}

ALP catalyzes the hydrolysis of various orthophosphoric monoesters to yield phosphate and the corresponding alcohols/ phenols. As one of the substrates of ALP, AAP can be converted to AA and phosphate. When AAP or ALP was added into the CD/ $\mathrm{Fe}(\mathrm{III})$ system, no obvious change in the fluorescence intensity was observed (Fig. 4A), whereas, the strong fluorescence signal was restored with the addition of both AAP and ALP to the CD/ $\mathrm{Fe}(\mathrm{III})$ system. Besides, the related fluorescence emission spectra are also displayed (Fig. S6 $\dagger$ ). Based on the above analysis, the enzymatically produced AA switches on the fluorescence of the CDs and a novel fluorescence assay for ALP can be developed.

The experimental conditions including the amount of iron ions, the amount of AAP, $\mathrm{pH}$ and the incubation time were optimized and the results are listed in Fig. S7.† Under the optimal conditions, the sensing performance of this system for the quantitative analysis of ALP was investigated by adding varying concentrations of ALP. As displayed in Fig. 4B, the fluorescence peak at $412 \mathrm{~nm}$ increased gradually with the increase of the amount of ALP from 0 to $1000 \mathrm{U} \mathrm{L}^{-1}$. A good linear fitting curve was obtained between the relative fluorescence intensity and ALP concentration from 5 to $200 \mathrm{U} \mathrm{L}^{-1}$ (Fig. 4C). The limit of detection for ALP was estimated to be 0.8 $\mathrm{U} \mathrm{L}^{-1}$ (with a signal-to-noise ratio of 3), which is comparable to those based on carbon dots reported recently (Table S2 $\dagger$ ). The results demonstrate that the proposed assay provides a sensitive platform for ALP detection. In order to evaluate the selectivity of the CD-based "switch-on" fluorescent probe for ALP, the effect of the proteins and anions possibly coexisting with ALP was investigated. As shown in Fig. 4D, the presence of GOx, lysozyme and trypsin result in slight fluorescence enhancements and no obvious fluorescence change is observed in the presence of $\mathrm{LDH}, \mathrm{GAL}$, thrombin, TYR, $\mathrm{Cl}^{-}$and $\mathrm{HCO}_{3}{ }^{-}$. However, the addition of ALP leads to a sharp increase of fluorescence intensity, indicating a good selectivity for the ALP response.

To test the applicability of the proposed assay to real human serum samples containing a variety of proteins and other contaminants, the assay for ALP in human serum was carried out. It is worth mentioning that the interference of GSH and Cys
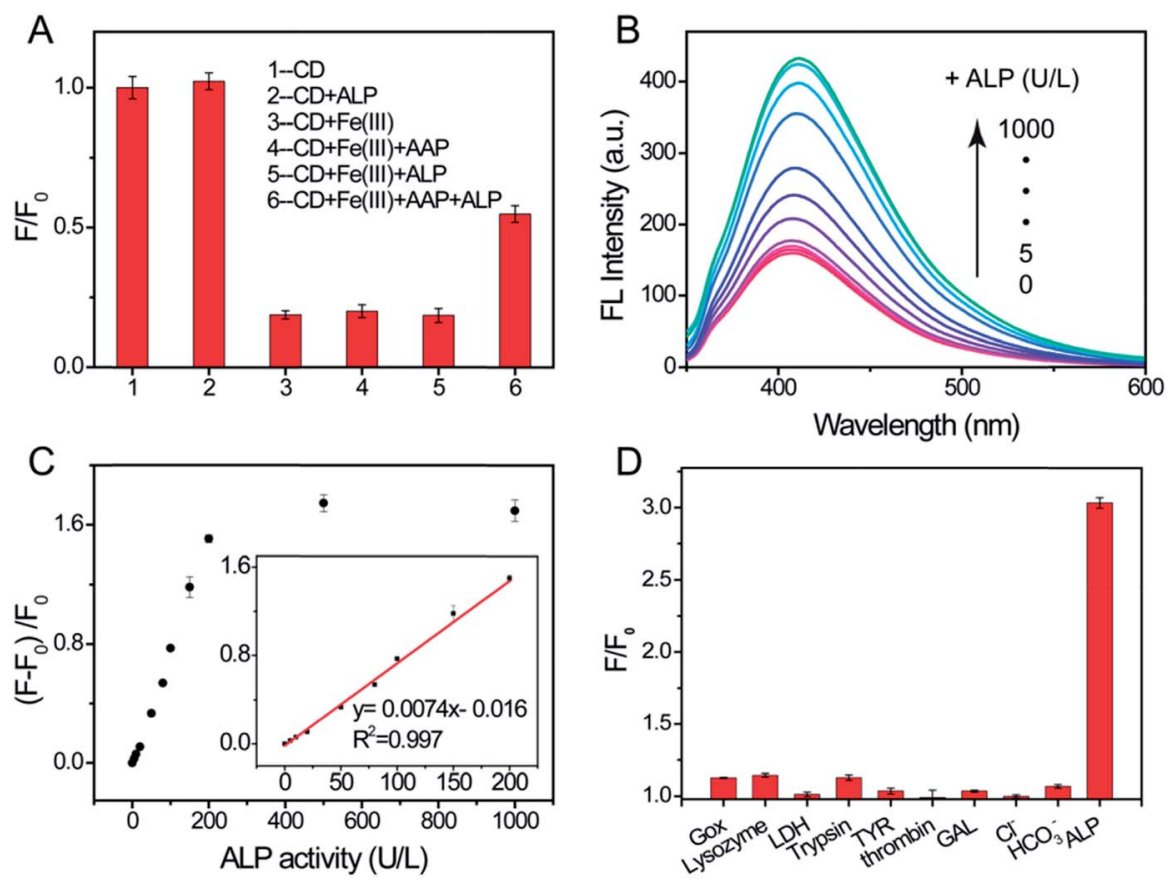

Fig. 4 (A) The fluorescence response of the $C D$ dispersion in the presence of different substances. (B) The emission spectra of the mixture containing CDs $\left(1 \mu \mathrm{L} \mathrm{mL}^{-1}\right)$, Fe(III) $(300 \mu \mathrm{M})$, and AAP $(50 \mathrm{mM})$ and an increasing amount of ALP from 0 to $1000 \mathrm{UL}^{-1}$. (C) The relative fluorescence intensity of the mixture versus ALP activity. Inset: the dependence of $\left(F_{0}-F\right) / F_{0}$ on ALP activity within the range of $5-200 \cup L^{-1}$. (D) Selectivity of the assay for ALP $\left(1000 \mathrm{U} \mathrm{L}^{-1}\right)$ compared to other proteins $\left(2000 \mathrm{U} \mathrm{L}^{-1}\right)$ and anions $(100 \mathrm{mM})$. 
in the serum samples can be eliminated by adding NEM (0.3 $\mathrm{mM})$, which is a scavenger that specifically reacts with GSH and Cys. ${ }^{41}$ Three serum samples with the addition of different concentrations of ALP were detected by the proposed method (Table $\mathrm{S} 3 \dagger$ ). The results indicate that our assay provides acceptable recoveries in the range of $93 \%$ to $110 \%$, suggesting the negligible influence from complex biological media on ALP sensing and the potential of the "switch-on" fluorescent probe for ALP detection in real biological samples.

\section{Human IgG detection}

Inspired by the successful and extensive application of ALP in the immunoassay, the highly selective and sensitive ALP sensing system has great potential to be extended to an ALPlabeled immunoassay for a target antigen. Fig. 5A schematically illustrates the recognition principle of the proposed fluorescence immunoassay, which has been used to detect human IgG (a model target). The anti-human IgG antibody and ALPlabeled anti-human IgG antibody were employed as capture and detection antibodies, respectively. Meanwhile, AAP was chosen as the substrate and ALP was used as the readout enzyme. After the formation of a sandwich-type immunocomplex in the presence of human IgG, the ALP bound on the polystyrene microwells triggers the AA-mediated fluorescence recovery of the CDs. Therefore, a fluorescence sandwich immunoassay can be established and developed.

The degree of fluorescence recovery is directly related to the concentration of the target antigen (Fig. 5B). A CD-based "switch-on" fluorescence immunoassay platform was developed where a serial increase of the target molecule resulted in different gradients of fluorescence intensity. The relative fluorescence intensity is proportional to the logarithm of the human IgG concentration in a linear range from 40 to $4000 \mathrm{ng}$ $\mathrm{mL}^{-1}$ (Fig. 5C). The limit of detection is $150 \mathrm{pg} \mathrm{mL} \mathrm{m}^{-1}$. As a comparison, the human IgG ELISA kit provided a LOD of $6 \mathrm{ng}$ $\mathrm{mL}^{-1}$, which has a lower sensitivity than that obtained by our "switch-on" fluorescence immunoassay. Additionally, the selectivity of the constructed immunoassay was evaluated. As depicted in Fig. $\mathrm{S} 8, \uparrow$ there is no significant interference observable from rabbit IgG, goat IgG and mouse IgG. However, this strategy could be applied to various target molecules simply by using different antigen-antibody pairs.

Human IgG is not only a widely used model antigen but also a biomarker of autoimmune diseases. Quantitative measurement of its presence in serum is highly valuable to the diagnosis of diseases and to the monitoring of treatment responses. ${ }^{42}$ In clinic, the amount of IgG in serum ranges from $7-16.5 \mathrm{mg} \mathrm{mL}^{-1}$ for healthy adults and that of patients with systemic

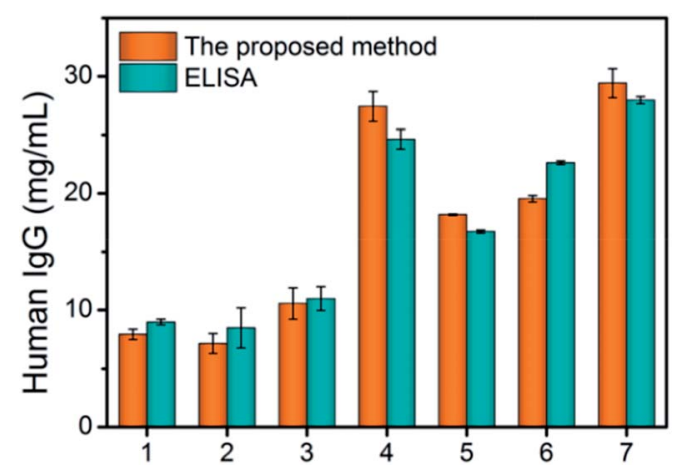

Fig. 6 The detection of human IgG in 7 serum samples using our method and ELISA kits. Serum samples of no. 1-3 were from normal adults; serum samples of no. 4-7 were from the patients with SLE.
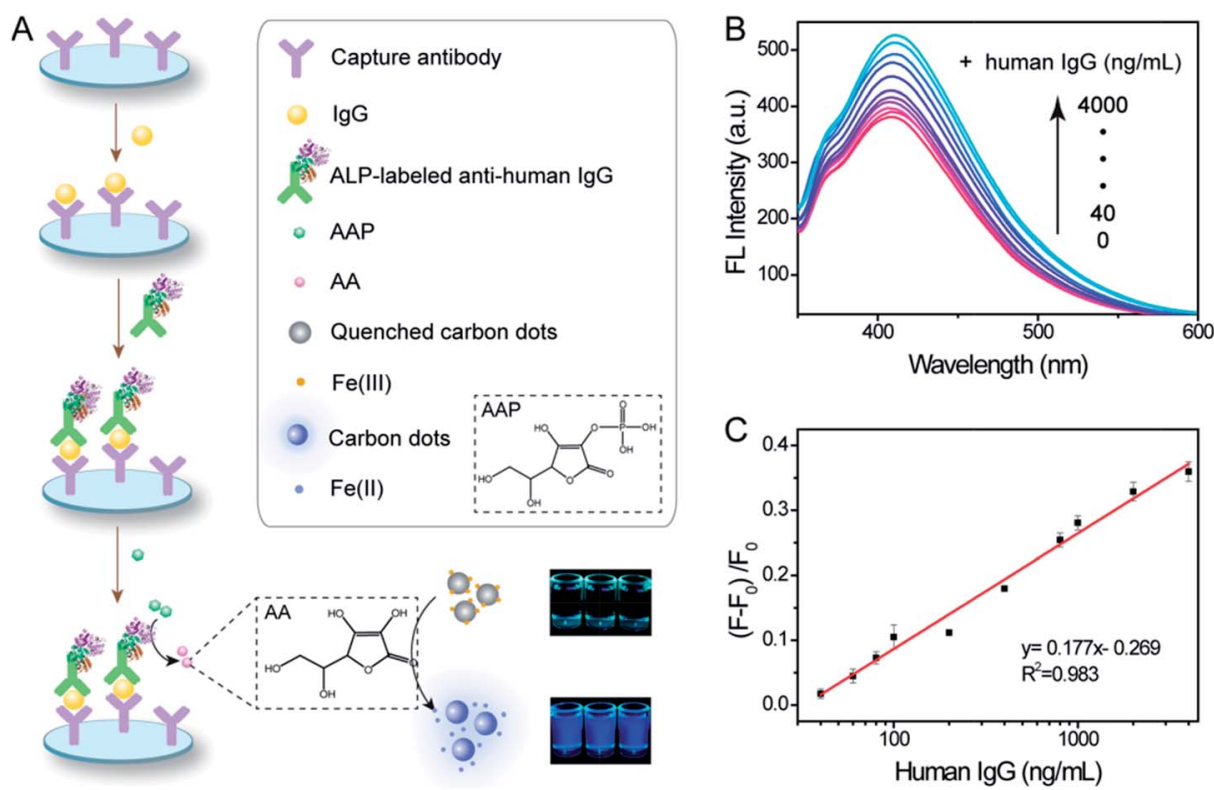

Fig. 5 (A) The switch-on fluorescence detection of human IgG through sandwich-type immunorecognition. (B) The recovered fluorescence of the CDs (that are quenched by Fe(III)) in the presence of different concentrations of human IgG. $(C)$ The linear fitting curve between $\left(F_{0}-F\right) / F_{0}$ and human IgG concentration . 
autoimmune diseases is higher. ${ }^{43}$ To demonstrate the reliability and clinical application potential of the assay, sera from 7 donors ( 3 healthy donors and 4 SLE patients) were assayed with either standard ELISA or the proposed CD-based sensing system. As demonstrated in Fig. 6, the IgG detection results from these two methods show highly consistent values and they were not considered statistically significant ( $P>0.1$ for $t$-tests). It can be concluded that the results have no significant difference. The result suggests that the proposed assay provides a potential alternative for early diagnosis and monitoring treatment responses.

\section{Conclusion}

In summary, a versatile CD-based platform modulated by a chemical redox "switch-on" fluorescence assay was presented, using lotus leaves as a raw material. This design was based on the phenomenon that the fluorescence of the prepared CDs was specifically quenched by $\mathrm{Fe}(\mathrm{III})$. The flexibility of this strategy has allowed us to quantify Fe(III), AA or ALP and detect human IgG in serum samples. The CDs prepared in this work possess many advantages, for example, they are low-cost and have high photostability and excellent biocompatibility, thus they are expected to be used in assays in vivo. The designed assay shows good specificity, sensitivity and capability of application to routine practice in clinic. Meanwhile, we envision that this "switch-on" fluorescence sensing platform is not limited to the above applications and has the potential to detect a variety of targets for biological analysis, early diagnosis, and treatment monitoring.

\section{Conflicts of interest}

There are no conflicts to declare.

\section{Acknowledgements}

This work was supported by financial support from the International Science \& Technology Cooperation Program of China (No. 2015DFR40460), National Natural Science Foundation under Grant No. 81402896 and No. 81573390, and the National High-Tech Research and Development Program of China (863 Program) under Grant No. 2014AA022303.

\section{Notes and references}

1 W. C. Chan and S. Nie, Science, 1998, 281, 2016-2018.

2 J. M. Nam, C. S. Thaxton and C. A. Mirkin, Science, 2003, 301, 1884-1886.

3 P. Cohen, Trends Biochem. Sci., 2000, 25, 596-601.

4 M. B. Yaffe, Nat. Rev. Mol. Cell Biol., 2002, 3, 177-186.

5 L. N. Johnson and R. J. Lewis, Chem. Rev., 2001, 101, 22092242.

6 S. G. Julien, N. Dube, S. Hardy and M. L. Tremblay, Nat. Rev. Cancer, 2011, 11, 35-49.

7 J. E. Coleman, Annu. Rev. Biophys. Biomol. Struct., 1992, 21, 441-483.
8 J. P. Lalles, Nutr. Rev., 2014, 72, 82-94.

9 Y. Kuang, J. Shi, J. Li, D. Yuan, K. A. Alberti, Q. Xu and B. Xu, Angew. Chem., Int. Ed., 2014, 53, 8104-8107.

10 M. C. L. Yeung and V. W. W. Yam, Chem. Sci., 2013, 4, 29282935.

11 J. A. Lorente, H. Valenzuela, J. Morote and A. Gelabert, Eur. J. Nucl. Med., 1999, 26, 625-632.

12 P. Colombatto, A. Randone, G. Civitico, J. Monti Gorin, L. Dolci, N. Medaina, F. Oliveri, G. Verme, G. Marchiaro, R. Pagni, P. Karayiannis, H. C. Thomas, G. Hess, F. Bonino and M. R. Brunetto, J. Viral Hepatitis, 1996, 3, 301-306.

13 P. Colombatto, A. Randone, G. Civitico, G. Monti Gorin, L. Dolci, N. Medaina, G. Calleri, F. Oliveri, M. Baldi, G. Tappero, R. Volpes, E. David, G. Verme, A. Smedile, F. Bonino and M. R. Brunetto, J. Viral Hepatitis, 1997, 4(suppl. 1), 55-60.

14 M. F. Hoylaerts, L. Ding, S. Narisawa, S. Van Kerckhoven and J. L. Millan, Biochemistry, 2006, 45, 9756-9766.

15 V. B. Paragas, Y. Z. Zhang, R. P. Haugland and V. L. Singer, J. Histochem. Cytochem., 1997, 45, 345-357.

16 K. P. Top, G. Hatleberg, K. N. Berggren, D. Ryan, C. Kemper, R. P. Haugland and W. F. Patton, Electrophoresis, 2001, 22, 896-905.

17 H. Duan, D. Wang and Y. Li, Chem. Soc. Rev., 2015, 44, 57785792.

18 J. Deng, W. Ma, P. Yu and L. Mao, Anal. Chem., 2015, 87, 6958-6965.

19 S. Y. Lim, W. Shen and Z. Gao, Chem. Soc. Rev., 2015, 44, 362381.

20 V. Strauss, J. T. Margraf, C. Dolle, B. Butz, T. J. Nacken, J. Walter, W. Bauer, W. Peukert, E. Spiecker, T. Clark and D. M. Guldi, J. Am. Chem. Soc., 2014, 136, 17308-17316.

21 G. E. LeCroy, S. K. Sonkar, F. Yang, L. M. Veca, P. Wang, K. N. Tackett, J. J. Yu, E. Vasile, H. Qian, Y. Liu, P. G. Luo and Y. P. Sun, ACS Nano, 2014, 8, 4522-4529.

22 Y. Wu, P. Wei, S. Pengpumkiat, E. A. Schumacher and V. T. Remcho, Anal. Chem., 2015, 87, 8510-8516.

23 Z. S. Qian, L. J. Chai, Y. Y. Huang, C. Tang, J. J. Shen, J. R. Chen and H. Feng, Biosens. Bioelectron., 2015, 68, 675680.

24 G. Li, H. Fu, X. Chen, P. Gong, G. Chen, L. Xia, H. Wang, J. You and Y. Wu, Anal. Chem., 2016, 88, 2720-2726.

25 C. Tang, Z. Qian, Y. Huang, J. Xu, H. Ao, M. Zhao, J. Zhou, J. Chen and H. Feng, Biosens. Bioelectron., 2016, 83, 274280.

26 C. C. Huang, C. T. Chen, Y. C. Shiang, Z. H. Lin and H. T. Chang, Anal. Chem., 2009, 81, 875-882.

27 Z. Zhou, Y. Du and S. Dong, Biosens. Bioelectron., 2011, 28, 33-37.

28 X. L. Hu, X. M. Wu, X. Fang, Z. J. Li and G. L. Wang, Biosens. Bioelectron., 2016, 77, 666-672.

29 Z. Qian, L. Chai, C. Tang, Y. Huang, J. Chen and H. Feng, Anal. Chem., 2015, 87, 2966-2973.

30 S. Liu, J. Tian, L. Wang, Y. Luo, J. Zhai and X. Sun, J. Mater. Chem., 2011, 21, 11726-11729.

31 Y. Yang, J. Cui, M. Zheng, C. Hu, S. Tan, Y. Xiao, Q. Yang and Y. Liu, Chem. Commun., 2012, 48, 380-382. 
32 D. Sun, R. Ban, P. H. Zhang, G. H. Wu, J. R. Zhang and J. J. Zhu, Carbon, 2013, 64, 424-434.

33 S. Li, Y. Li, J. Cao, J. Zhu, L. Fan and X. Li, Anal. Chem., 2014, 86, 10201-10207.

34 X. C. Sun, C. Bruckner and Y. Lei, Nanoscale, 2015, 7, 1727817282.

35 Y. Q. Dong, H. C. Pang, H. B. Yang, C. X. Guo, J. W. Shao, Y. W. Chi, C. M. Li and T. Yu, Angew. Chem., Int. Ed., 2013, 52, 7800-7804.

36 A. Jaiswal, S. S. Ghosh and A. Chattopadhyay, Chem. Commun., 2012, 48, 407-409.
37 B. Shi, Y. Su, L. Zhang, M. Huang, R. Liu and S. Zhao, ACS Appl. Mater. Interfaces, 2016, 8, 10717-10725.

38 Z. Gao, L. B. Wang, R. X. Su, R. L. Huang, W. Qi and Z. M. He, Biosens. Bioelectron., 2015, 70, 232-238.

39 J. H. Lin, Y. C. Yang, Y. C. Shih, S. Y. Hung, C. Y. Lu and W. L. Tseng, Biosens. Bioelectron., 2016, 77, 242-248.

40 J. J. Mullay, J. Am. Chem. Soc., 1985, 107, 7271-7275.

41 J. Yuan, Y. Cen, X. J. Kong, S. Wu, C. L. Liu, R. Q. Yu and X. Chu, ACS Appl. Mater. Interfaces, 2015, 7, 10548-10555.

42 R. C. Cunningham, Blood, 2010, 116, 7-15.

43 D. C. Heiner, Am. J. Med., 1984, 76, 1-6. 